\title{
Chamas for Change: Redevelopment of Community Health Volunteer Curriculum for a Group-Based Maternal-Child Peer Support and Microfinance Care Model in Western Kenya
}

\author{
Grace Rushton BS, ${ }^{1}$ Julia Jerono Songok MMBchB, ${ }^{2,}{ }^{3}$ Anjellah Jumah, ${ }^{2}$ \\ Justus E. Ikemeri BSc, ${ }^{2}$ Laura Ruhl MD MPH ${ }^{1,2,3}$ \\ ${ }^{1}$ Indiana University School of Medicine, Indianapolis, IN; ${ }^{2}$ Academic Model Providing \\ Access to Healthcare, Eldoret, Kenya; ${ }^{3}$ Department of Child Health \& Paediatrics, \\ College of Health Science, Moi University, Eldoret, Kenya
}

Background: Maternal mortality continues to be the leading cause of death for women of child-bearing age in Kenya. Chamas for Change builds on longstanding cultural practices as a community-based peer support model for pregnant and breast-feeding mothers. Through medical and social education, microfinancing for economic empowerment, and a culture of community accountability, Chamas enables women to advocate for their health and improve health outcomes for their children. With significant success over the past eight years and a recently completed cluster randomized control trial (RCT), the Chamas for Change team is looking to expand the scope of Chamas to additional counties in Kenya and beyond.

Project: To prepare for this transition to scale, the Chamas flipchart curriculum was redeveloped to include additional education topics and restructured to make each of the three cycles (or years) more cohesive and structured. To investigate new topics, brainstorming was conducted within the Maternal, Newborn, and Child Health (MNCH) team. Community Health Volunteers (CHVs) and implementation leads for the program were surveyed and a previously completed focus group discussion with Chamas members was analyzed. Lastly, additional topics were gathered from existing child development and adolescent reproductive health curricula through AMPATH.

Results: Content for new medical and social topics was developed and integrated with existing topics. A total of 24 medical and 24 social topics were established for each of the two initial cycles of the program. Each session includes a reflection, time for socializing and activities, health and social education content, and homework to prompt further reflection.

Conclusions and Impact: This refined flipchart will help to address demands from Chama mothers who are seeking a more robust education, while also further standardizing and broadening the curriculum for CHVs. It will additionally 
allow the Chamas team to prepare to provide a quality toolkit to international partners in the future with the hope of improving $\mathrm{MNCH}$ outcomes worldwide. 